\title{
Association between the fatigue and sleep quality of kidney transplant recipients: The mediating role of rumination
}

ping zhang

Second Hospital,Cheeloo College of Medicine,Shandong University

Xiao-Li Liu

Second Hospital,Cheeloo College of Medicine,Shandong University

Xin Li

Second Hospital,Cheeloo College of Medicine,Shandong University

Jing-Hua Yang

Second Hospital,Cheeloo College of Medicine,Shandong University

Rong-Mei Zhang ( $\sim 15153169039 @ 126 . c o m$ )

Second Hospital,Cheeloo College of Medicine,Shandong University

\section{Research Article}

Keywords: Kidney transplantation, Fatigue, Sleep quality, Rumination

Posted Date: November 30th, 2021

DOl: https://doi.org/10.21203/rs.3.rs-959749/v1

License: (9) This work is licensed under a Creative Commons Attribution 4.0 International License. Read Full License 


\section{Abstract \\ Background}

Sleep disorders persist in renal transplant patients and are an important determinant of low quality of life. This study aims to investigate the mediating role of rumination in the relationship between fatigue and sleep quality in kidney transplant recipients.

\section{Method}

A descriptive cross-sectional research design was implemented from April to September 2021.A general information questionnaire, Pittsburgh Sleep Quality Index (PSQI), Multidimensional Fatigue Inventory (MFI-20), and Rumination Response Scale (RRS) were used to investigate 192 kidney transplant patients who were re-examined at the outpatient departments of three first-class hospitals in Jinan, Shandong Province, China. The Pearson's correlation coefficient and mediation analyses were used for the statistical and descriptive statistics analyses.

\section{Results}

The prevalence of sleep disorders among kidney transplant recipients was $19.3 \%$. With rumination as a mediator, fatigue indirectly affected the patients' sleep quality. This indirect effect was $0.10(95 \%$ confidence interval: $0.154,0.419)$. Medical staff should focus on fatigue symptoms and rumination of patients to enhance sleep quality of kidney transplant recipients.

\section{Conclusions}

The incidence of sleep disorders after renal transplantation was high.Rumination exerts a mediating role between fatigue and sleep quality.

\section{Background}

Renal transplantation is an ideal method for treating end-stage renal disease and improving patients' quality of life(1). However, although patients' quality of life is somewhat improved after the transplantations, they are still affected by certain factors, including the source of transplanted kidney, living environment, economic status, postoperative immune rejection, and various other complications. These factors may cause adverse emotions in postoperative patients, which are detrimental to their health and can seriously reduce their quality of life(2-4).

The incidence of sleep disorders, which are indicators of a low quality of life, in patients with chronic kidney disease and end-stage renal disease is very high $(5,6)$. Renal transplantation is the preferred 
kidney replacement therapy for patients with stage 5 chronic kidney disease(7). This therapy is more effective at prolonging patients' life expectancy and improving their health-related quality of life, when compared to dialysis(8). However, studies indicate that patients' sleep disorders (e.g., restless leg syndrome, sleep hypopnea, and sleep apnea) were not alleviated after kidney transplants. In fact, several patients had persistent or new sleep disorders $(2,9)$, and their quality of sleep was lower than that of general patients(10). Hasanzamani et al. (2020) recorded patients' sleep quality before, three months after, and six months, after their renal transplants, and the data revealed that patients' incidence of sleep disorders were $37.5 \%, 37.5 \%$, and $20.0 \%$, respectively(11).Furthermore, Xie et al. (2018) determined that the incidence of sleep disorders among Chinese kidney transplant recipients was $29.2 \%(10)$. Sleep disorders can lead to daytime sleepiness and a decline in life function, which is not only harmful to patients' physical, psychological, and social health but also negatively affects their cognitive functioning, behavior, and attention $(3,6,9)$.Therefore, it is imperative to identify the risk factors associated with poor sleep quality and the rehabilitation mechanisms that can improve patients' quality of daily life.

Fatigue is a subjective feeling of being overwhelmingly and continuously exhausted. Furthermore, it is a decline of normal physical and mental strength levels that is closely related to various acute and chronic diseases. Fatigue negatively affects patients' self-care abilities and their quality of life in physiological, social, and other aspects $(3,6,12)$. Although renal transplants significantly improve the quality of life of patients with end-stage renal disease, related reports revealed that $39-59 \%$ of the recipients still experience a certain degree of fatigue $(13,14)$.Fatigue, hypertension, and serious hair loss are the three most painful symptoms in Chinese renal transplant recipients(15). Additionally, several studies indicate that behavioral-psychosocial factors are directly related to fatigue. These factors often influence each other; for example, depressive symptoms and sleep disorders can aggravate fatigue, while fatigue can cause and aggravate depression and sleep disorders. Bossola, Pepe, and Vulpio (2016) discovered that fatigue and negative emotions (such as depression) are factors that strongly influence the sleep disorders of patients who received renal transplants(16). Another study suggested that sleep disorders are prominent risk factors of fatigue(14), which denotes the close association between fatigue and sleep disorders.

Rumination is a kind of negative cognitive style that occurs while encountering negative events. It entails continuous negative thoughts about the events themselves and the possible ensuing consequences, instead of constructive problem-solving actions. This negative cognition style is associated with increases in negative emotions and the decreased inhibitory control of negative information. Subsequently, this results in stable negative emotional patterns that lead to adverse health consequences, such as impaired sleep and weakened health $(17,18)$. The microanalysis model of insomnia indicates that excessive wakefulness is the main cause of insomnia(19).This wakefulness is characterized by bedtime arousal, which consists of cognitive and physical arousal; cognitive arousal is defined as uncontrollable invasive cognition(20). Therefore,rumination-as a form of invasive cognitioncauses selective attention concerned with perceived negative cues before going to bed, which then stimulates more cognitive arousal and further delays the onset of sleep(21). Accordingly, previous studies have confirmed that rumination can cause insomnia(22), and that the higher the level of rumination, the 
more serious the insomnia $(23,24)$. Other studies suggest that rumination may be a mediating mechanism between stressful events and insomnia $(17,22)$. Further research indicated that there was a significant positive correlation between fatigue and rumination and that fatigue may indirectly affect depression through rumination(15). However, few studies are concerned with the sleep quality of renal transplant patients in China, and it is unclear whether rumination plays a mediating role between fatigue and sleep quality among these patients. Thus, this study aims to explore the association between fatigue and sleep quality and to investigate the mediating effect of rumination.

\section{Methods}

All methods are implemented in accordance with the guidelines and regulations.

\section{Study design,participants and data collection}

This study aimed to investigate the prevalence of sleep disorders among kidney transplant patients who were re-examined at outpatient departments. Furthermore, it explored the possible mediating role of rumination between fatigue and sleep quality. A convenience sample of 192 kidney transplant recipients based cross-sectional study who were re-examined at outpatient departments were recruited in three firstclass hospitals from April to September 2021 in Jinan, Shandong Province, China. The participant inclusion criteria were as follows: (a) received a kidney transplant a year or more ago, (b) aged 18 or above, and (c) possesses sound Chinese language and reading skills. The exclusion criteria were: (a) patients suffering from cognitive impairment or a psychotic illness (b) kidney transplant patients with severe heart, liver, or other organ diseases.

Before the implementation of the questionnaire, consent was obtained from the hospital ethics committee, and communications were established with the outpatient doctors and relevant responsible nurses. The questionnaire consisted of four parts: general demographic information, fatigue, rumination, and sleep quality. The data of all patients were collected by face-to-face interviews in separate rooms. Additionally, informed consent was obtained from all patients, and their information was collected anonymously and kept confidential.

\section{Measures}

\section{Demographic questionnaire}

Demographic data were compiled by researchers through literature reviews and clinical practice, which included patients' gender, age, educational status, marital status, economic status (CNY/month), time since kidney transplantation and current creatinine $(\mathrm{Cr})$.

\section{Multidimensional Fatigue Inventory (MFI-20)}


A total of $20 \mathrm{MFI}$ items were used to evaluate patients' fatigue; the items were developed by Smets, Garssen, Bonke and De Haes (1995) (25)and translated into Chinese by Chung et al. (2014)(26). The MFI20 included five dimensions pertaining to general fatigue, physical fatigue, reduced motivation, mental fatigue, and reduced activity. Each item was scored on a five-point Likert scale ranging from 1 "absolutely not" to 5 "absolutely". The participants' total scores ranged from 20 to 100; the higher their score, the more fatigued they were. The Cronbach's a of the MFI-20 was 0.772 in our study.

\section{Rumination Response Scale (RRS)}

The RRS was compiled by Nolen-Hoeksema and Morrow (1991)(27).The Chinese version, was revised by Han and Yan (2009)(28) and adopted in this study to measure rumination; it normally exhibits a Cronbach's a of 0.91 . The scale consisted of 22 items that were rated on a four-point Likert scale ranging from 1 "never" to 4 "almost always." The patients' total scores ranged from 22 to 88 ; higher scores indicate higher rumination levels. The Cronbach's a in this study was 0.924.

\section{Pittsburgh Sleep Quality Index (PSQI)}

The PSQI was developed by Buysse, Reynolds, Monk, Berman \& Kupfer (1989) (29)and has been widely used to assess healthy people and patients with sleep or mental disorders in China; it exhibits good reliability and validity $(30,31)$. The PSQI comprises 19 self-reported questions, and each item is scored from $0-3$. The patients' total scores were between $0-21$; higher scores indicate worse sleep quality. In this study, a total score of PSQI > 7 indicated poor sleep quality(32). The Cronbach's a was 0.850 in this study.

\section{Statistical analysis}

This study used the SPSS version 23.0 (IBM Corp, Armonk, NY, United States) for data analysis. The characteristics of and differences in sleep quality were described with a descriptive analysis, independent t-test, and the one-way analysis of variance (ANOVA). The Pearson's correlation coefficient ( $r$ ) was calculated to assess the relationships between fatigue, rumination, and sleep quality. The mediating effect of rumination was examined with the PROCESS macro (Model 4) produced by Hayes (Hayes, 2013). All variables were standardized before the data analysis commenced, and demographic variables were controlled during the mediation analysis process. Furthermore, bootstrapping was used to test the mediating effect of rumination by setting a sample size of 5000 and a confidence interval $(\mathrm{Cl})$ of $95 \%$. It revealed that the mediating model was established if the $95 \% \mathrm{Cl}$ of indirect effect did not contain zero. Additionally, a value of $\mathrm{P}<0.05$ was considered statistically significant for all data processing.

\section{Results}

\section{Sample characteristics}

This study was conducted with 192 renal transplant patients who were re-examined at outpatient departments in China. Table 1 displays the patients' demographics, disease characteristics, and a 
distribution of their sleep quality. The patients' average age was 44 years ( $S D=10.65$, range $20-75$ years). The majority of the sample participants were male (69.3\%), married (91.7\%), and accepted middle school (38.5\%). Only $43.2 \%$ of the participants' income was between 2000 and $4000 \mathrm{CNY} /$ month. Furthermore, most patients had received their kidney transplants more than five years ago, and $70.3 \%$ of them exhibited normal creatinine levels. Additionally, statistically significant differences in sleep quality were found between sex, age, and economic status (CNY/month) $[(t=-3.194, P=0.002 ; F=3.392, P=$ $0.036 ; \mathrm{F}=3.442, \mathrm{P}=0.034$, respectively)]. 
Table 1

Sample characteristics and the distribution of sleep quality $(\mathrm{N}=192)$

\begin{tabular}{|c|c|c|c|c|}
\hline \multirow[t]{2}{*}{ Variables } & \multirow{2}{*}{$\begin{array}{l}\text { Number of people } \\
(\mathrm{N}=192)(\%)\end{array}$} & \multicolumn{3}{|c|}{ sleep quality( $\mathrm{N}=192)$} \\
\hline & & Mean(SD) & $t / F$ & $P$ \\
\hline Sex & & & -3.194 & 0.002 \\
\hline Male & 133(69.3) & $4.99(2.53)$ & & \\
\hline Female & $59(30.7)$ & $6.31(2.82)$ & & \\
\hline \multicolumn{5}{|l|}{ Age } \\
\hline $18 \sim 40$ & $82(42.7)$ & $4.95(2.35)$ & 3.392 & 0.036 \\
\hline $41 \sim 59$ & $94(49.0)$ & $5.55(2.80)$ & & \\
\hline$\geq 60$ & $16(8.3)$ & $6.75(3.26)$ & & \\
\hline Marital status & & & 1.231 & 0.220 \\
\hline Married & 176(91.7) & $6.19(2.90)$ & & \\
\hline Unmarried & $16(8.3)$ & $5.32(2.67)$ & & \\
\hline Educational status & & & 1.347 & 1.261 \\
\hline Primary school or under & $18(9.4)$ & $5.61(2.12)$ & & \\
\hline Middle school & $74(38.5)$ & $5.35(2.74)$ & & \\
\hline High school & $50(26.0)$ & $5.92(3.10)$ & & \\
\hline College or above & $50(26.0)$ & $4.86(2.29)$ & & \\
\hline Economic status (CNY/month) & & & 3.442 & 0.034 \\
\hline$<2000$ & $68(35.4)$ & $5.57(2.67)$ & & \\
\hline $2000 \sim 4000$ & $83(43.2)$ & $5.72(2.66)$ & & \\
\hline$>4000$ & $41(21.4)$ & $4.44(2.62)$ & & \\
\hline \multicolumn{5}{|c|}{$\begin{array}{l}\text { Time since kidney Transplantation } \\
\text { (years) }\end{array}$} \\
\hline$\leq 5$ & $64(33.3)$ & $5.67(2.70)$ & 1.006 & 0.316 \\
\hline$>5$ & $129(66.7)$ & $5.26(2.68)$ & & \\
\hline Current $\mathrm{Cr}$ & & & -0.084 & 0.993 \\
\hline normal & 135(70.3) & $5.39(2.75)$ & & \\
\hline
\end{tabular}




\begin{tabular}{|lll|}
\hline Variables & $\begin{array}{l}\text { Number of people } \\
(\mathbf{N}=192)(\%)\end{array}$ & sleep quality $(\mathbf{N}=192)$ \\
\hline abnormal & $57(29.7)$ & $5.42(2.56)$ \\
\hline
\end{tabular}

\section{Correlational analyses}

Table 2 displays the means, SDs, and correlations of the main study variables. The means and SDs pertaining to the total scores of fatigue, rumination, and sleep quality were 46.49(11.37), 37.49(10.11), and 5.40(2.69), respectively. The Pearson product-moment correlations indicated that sleep quality was positively correlated with both fatigue $(r=0.394, P<0.001)$ and rumination $(r=0.418, P<0.01)$. Moreover, fatigue was also positively correlated with rumination $(r=0.368, P<0.001)$.

Table 2

Bivariate correlation among fatigue, rumination, and sleep quality

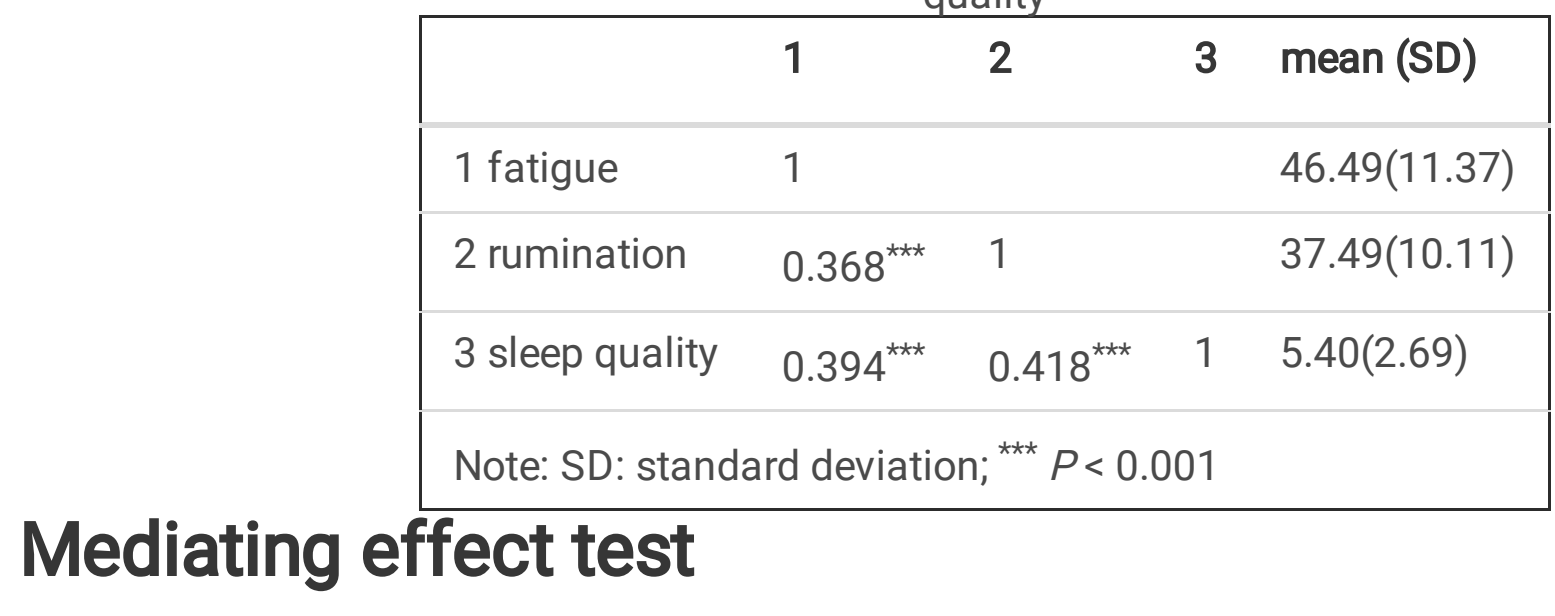

The results of the mediation analysis are displayed in Table 3. The data were standardized before the analysis of the mediating effect was conducted. After controlling for demographic variables, the results revealed that the total effect (path $c$ ) of fatigue on sleep quality was $0.37(P<0.001)$; fatigue was significantly associated with rumination $(\beta=0.35, p<0.001)$; and rumination was significantly associated with sleep quality $(\beta=0.29, P<0.001)$. Furthermore, even when rumination was controlled, the previously significant relationship between fatigue and sleep quality (direct effect) still existed $(\beta=0.27, P<0.001)$. Additionally, the bootstrap test results indicate that the $95 \% \mathrm{Cl}$ of rumination's indirect effect between fatigue and sleep quality did not include zero $(\mathrm{Cl}=0.154,0.419$; indirect effect $=0.10)$. It also revealed that the mediating effect was statistically significant, which indicates that rumination acts as a mediator between fatigue and sleep quality. The standardized mediating effect was 0.10 , and the mediating effect accounted for $27 \%$ of the total effect. Figure 1 displays the final mediating model. 
Table 3

Mediation of rumination between fatigue and sleep quality

\begin{tabular}{|c|c|c|c|c|c|c|c|c|}
\hline & $R^{2}$ & $F$ & $\begin{array}{l}\text { Coeff } \\
\text { icient }\end{array}$ & SE & $t$ & $P$ & LLCl & ULCI \\
\hline Outcome:Rumination & 0.164 & 9.177 & & & & & & \\
\hline Fatigue $^{a}$ & & & 0.35 & 0.067 & 5.198 & $<0.001$ & 0.218 & 0.485 \\
\hline Outcome: Sleep Quality & 0.299 & 15.853 & & & & & & \\
\hline Fatigue $\mathrm{c}^{\prime}$ & & & 0.27 & 0.066 & 3.998 & $<0.001$ & 0.134 & 0.396 \\
\hline Rumination $\mathrm{b}$ & & & 0.29 & 0.067 & 4.265 & $<0.001$ & 0.154 & 0.419 \\
\hline \multicolumn{9}{|c|}{$\begin{array}{l}\text { Note: SE:standard error; LLCI: lower level of confidence interval; ULCl: upper level of confidence } \\
\text { interval; a : path fatigue-rumination; b: path rumination-sleep quality; c': path fatigue }\end{array}$} \\
\hline \multicolumn{9}{|c|}{-sleep quality when rumination acting as a mediator (indirect effect). } \\
\hline
\end{tabular}

\section{Discussion}

Our results revealed that rumination assumes a significant mediating role between fatigue and sleep quality. Thus, the more tired kidney transplant recipients become, the more likely they are to ruminate, which leads to a decline in sleep quality. This study discovered a lower incidence of sleep disorders; only 19.3\% (defined as a PSQI score $>7$ ) in kidney transplant recipients than in the studies by Xie et al. (2018) (29.2\%, poor sleeper defined as PSQI >7)(10) and Reilly-Spong, Park and Gross (2013) (41\%, poor sleeper defined as PSQI >8)(33). Several factors may account for this difference, these include: these patients' transplants occurred more than one year ago, their renal functions have had time to recover, their sleep difficulties might be relieved, the sample size may be too small, the sample populations differ, and the data were collected from different cultural settings. Nevertheless, this finding indicates that although the patients' sleep difficulties have been alleviated to some extent after their kidney transplants, the overall poor sleep quality persists. Hasanzamani et al. (2020) also proved that renal transplants can improve patients' sleep conditions after six months(11). Generally speaking, patients still have sleeping disorders more than a year after their kidney transplants, and the prevalence is high.

Furthermore, the study findings suggested that there was a significant positive correlation between fatigue scores and sleep disorder scores. This implies that higher fatigue scores lead to higher sleep disorder scores and poor sleep quality; Rodrigue et al. (2011) concurred with this conclusion(34). Studies also discovered that fatigue, hypertension, and hair loss are the three most common symptoms among renal transplant recipients in China(35). Additionally, since the patients cannot engage in heavy physical activities due to their transplanted kidneys, they become more sedentary and less active, which leads to fatigue. However, this type of fatigue is different from the fatigue caused by normal activities since it causes apathy and discomfort in patients' limbs, which makes it difficult to fall asleep. However, fatigue 
not only affect quality of life, but also reduces patients' tolerance to immunosuppressants, which increases their incidence of immune rejection and risk of death(36). Additionally, previous studies have pointed out that the severity of fatigue is closely related to sleeping disorders, and poor sleep quality will aggravate the fatigue of patients, which eventually lead to a decline in the quality of life(37).

The study results further indicate that, in addition to directly affecting patients sleep quality, fatigue also indirectly affects sleep quality through rumination. The research confirmed that fatigue is positively correlated with rumination, while rumination is positively correlated with sleeping disorders. Very few studies have explored the relationship between fatigue and rumination, and only explored the effects of work-related fatigue in employees(38-40).Nevertheless, previous studies revealed several correlations: physical fatigue can lead to a decline in athletic ability (i.e., lack of muscle strength); cognitive fatigue can lead to a decline in cognitive ability (i.e., poor attention and sensitivity); and mental fatigue can lead to negative emotions or stress disorders $(41,42)$. Thus, fatigue is a complex concept, and different performance of fatigue can enhance patients' ruminant thinking levels, resulting in reduced sleep quality. $\mathrm{Li}$ et al. (2019) found that stressful life events can both directly and indirectly affect sleep quality through rumination(23). Furthermore, since stress can lead to the activation of the locus coeruleus noradrenaline (LC-NE) system and the hypothalamus pituitary adrenal (HPA) axis, it can increase excitability and aggravate existing difficulties in falling asleep $(43,44)$ According to Morin, Rodrgue, and Ivers' $(2003)$ insomnia-hyperactivity theory, cognitive arousal may play an important role in sleep disorders(45). Therefore, higher levels of rumination make people more prone to experiencing negative re-evaluations of the past, which increases their cognitive arousal and reduces sleep quality; thus supporting the "emotional flow" theory(46).

Diagnoses of renal failure and the subsequent kidney transplants are major life events that place strain on patients. Furthermore, since most patients will experience fear and then try to control their fear situation (anger), they may experience a "fight" response which will affect their sleep. Thus, the order of emotional change is fear-anger-happiness/sadness. Additionally, this result also supports the stress theory(47). Stress theory refers to the process of individual psychological adjustment and adaptation when faced with stress, whereas coping styles pertain to behavioral patterns that buffer and control an individual's response to stress stimuli during the coping process; this is aimed at solving or mediating an individual's emotions $(47,48)$.Positive coping styles directly affect an individual's compliance with disease treatment, which then affect their overall health status; negative coping styles are not conducive to patients' health outcomes $(47,49)$. Rumination is a type of negative coping style that exacerbates the severe decline of sleep quality. When engaging in ruminant thinking, individuals easily become caught up in memories of negative events, this leads to excessive fantasizing during which they lose track of time(50).Self-accusatory thoughts are common while ruminating and are focused on the individual's past mistakes and subsequent consequences. Individual's then question why their problems are unique to themselves which further aggravates their negative thoughts and behaviors. Therefore, the cycle of rumination is characterized by a focus on negative emotions which then increases insomnia inducing factors, maintenance factors of insomnia, and finally persistent symptoms of insomnia(44). 
This study demonstrated that rumination acts as a mediator between kidney transplant recipients' fatigue and their sleep quality. However, given that the causes of fatigue are related to the source of transplanted kidney(51), graft dysfunctions(34), and the use of immunosuppressants(13), improving patients' fatigue may not be very easy. Therefore, medical staff should employ effective measures that reduce patients' levels of rumination. Furthermore, rumination causes individuals to fixate on the negative aspects of events and the possible negative effects; this induces negative emotional experiences (e.g., loneliness and depression) that cause adverse health consequences, such as impaired sleep quality $(52,53)$. Accordingly, nurses should pay close attention to the emotional states and psychological characteristics of their patients. This includes practically caring about patients during daily nursing work, such as encouraging their families to visit, to correct patients' flawed perspectives over time. Meanwhile, the tendency of ruminating individuals to indulge in their own negative thought patterns and emotions increases their need for more social support to alleviate their inner pain. Therefore, nurses should provide the necessary social support for kidney transplant patients, such as strengthening communication with them and holding relevant informative lectures. Additionally, rumination interventions are very important. Previous studies have revealed that mindfulness therapy $(54,55)$, cognitive behavior interventions (56), and working memory training $(57,58)$ all play important roles in eliminating negative thinking and promoting positive thinking.

The study limitations relate to the use of a convenience sample of kidney transplant recipients located in one geographic area. This means that the representativeness and extrapolation efficiency of the conclusions are limited. Second, this study utilized a cross-sectional design to explore the effects of rumination on the relationship between fatigue and sleep quality of renal transplant patients. Consequently, this design cannot provide an accurate explanation for the causality between the two variables. Therefore, longitudinal studies or randomized controlled trials (RCTs) are necessary to confirm our results. Third, rumination was the only mediator considered in this study to determine the relationship between fatigue and sleep quality. However, other mediators may also affect the relationship between fatigue and sleep quality, including social support, family functions, anxiety, and depression. Future studies should include additional variables to comprehensively assess the impact of the results. Finally, the patients' conditions were assessed with a self-reported questionnaire, which may be biased.

\section{Conclusions}

In summary, our study found that a high incidence of sleep disorders and demonstrated that rumination in renal transplant patients function as a partial mediator between the dimensions of fatigue and sleep quality. According to our findings, fatigue related intervention strategies achieved marginal success, but rumination related interventions might lead to the greatest direct contribution toward preventing fatigue related sleep difficulties.

\section{Declarations}

\section{Acknowledgments}


We acknowledge the patients who participated in this study and the nursing colleagues in the second hospital of Shandong University for their assistance and cooperation.

\section{Disclosure}

This research received no external funding.

\section{Authors' contributions}

Reseach design,Ping Zhang and Rong-Mei Zhang;Formal analysis, Ping Zhang; Article quality and language polish, Xin Li and Jing-HuaYang;literature searches and analyses,Ping Zhang,Jing-HuaYang and Xin Li;Statistical analysis,Ping Zhang and Xiao-Li Liu;Writing-original draft,Ping Zhang,Rong-Mei Zhang and Xiao-Li Liu;Writing-review \& editing,Ping Zhang.

\section{Availability of data and materials}

The data that support the findings of this study are available from the corresponding author upon reasonable request.

\section{Ethical approval}

This study was reviewed and approved by the ethics committee of the Second Hospital,Cheeloo College of Medicine,Shandong University.All participants signed a written informed consent before the start of the study.

\section{Consent for publication}

Not applicable.

\section{Competing interests}

The authors declare no conflicts of interest.

\section{Author details}

${ }^{1}$ Department of Transplantation, Second Hospital,Cheeloo College of Medicine,Shandong University, Jinan, Shandong, China.

\section{References}

1. Kostro JZ, Hellmann A, Kobiela J, Skora I, Lichodziejewska-Niemierko M, Debska-Slizien A, et al. Quality of Life After Kidney Transplantation: A Prospective Study. Transplant Proc. 2016;48(1):50-4.

2. Burkhalter H, Brunner DP, Wirz-Justice A, Cajochen C, Weaver TE, Steiger J, et al. Self-reported sleep disturbances in renal transplant recipients. BMC Nephrol. 2013;14:220. 
3. De Pasquale C, Pistorio ML, Veroux M, Indelicato L, Biffa G, Bennardi N, et al. Psychological and Psychopathological Aspects of Kidney Transplantation: A Systematic Review. Front Psychiatry. 2020;11:106.

4. Griva K, Davenport A, Harrison M, Newman SP. Non-adherence to immunosuppressive medications in kidney transplantation: intent vs. forgetfulness and clinical markers of medication intake. Ann Behav Med. 2012;44(1):85-93.

5. Liu HX, Lin J, Lin XH, Wallace L, Teng S, Zhang SP, et al. Quality of sleep and health-related quality of life in renal transplant recipients. Int J Clin Exp Med. 2015;8(9):16191-8.

6. van Sandwijk MS, Al Arashi D, van de Hare FM, van der Torren JMR, Kersten MJ, Bijlsma JA, et al. Fatigue, anxiety, depression and quality of life in kidney transplant recipients, haemodialysis patients, patients with a haematological malignancy and healthy controls. Nephrol Dial Transplant. 2019;34(5):833-8.

7. Tkachenko D, Franke L, Peters L, Schiffer M, Zimmermann T. Dyadic Coping of Kidney Transplant Recipients and Their Partners: Sex and Role Differences. Front Psychol. 2019;10:397.

8. Liaveri PG, Dikeos D, Ilias I, Lygkoni EP, Boletis IN, Skalioti C, et al. Quality of sleep in renal transplant recipients and patients on hemodialysis. J Psychosom Res. 2017;93:96-101.

9. Eslami AA, Rabiei L, Khayri F, Rashidi Nooshabadi MR, Masoudi R. Sleep quality and spiritual wellbeing in hemodialysis patients. Iran Red Crescent Med J. 2014;16(7):e17155.

10. Xie J, Wang L, Tang X, Yi Y, Ding S, Yan J, et al. Sleep Quality and Related Psychosocial Variables Among Renal Transplant Patients. Transplant Proc. 2018;50(10):3426-33.

11. Hasanzamani B, Pourranjbar E, Rezaei Ardani A. Comparing Sleep Quality in Patients Before and After Kidney Transplantation. Iran J Kidney Dis. 2020;14(2):139-44.

12. Samarehfekri A, Dehghan M, Arab M, Ebadzadeh MR. Effect of Foot Reflexology on Pain, Fatigue, and Quality of Sleep after Kidney Transplantation Surgery: A Parallel Randomized Controlled Trial. Evid Based Complement Alternat Med. 2020;2020:5095071.

13. Chan W, Bosch JA, Jones D, Kaur O, Inston N, Moore S, et al. Predictors and consequences of fatigue in prevalent kidney transplant recipients. Transplantation. 2013;96(11):987-94.

14. Goedendorp MM, Hoitsma AJ, Bloot L, Bleijenberg G, Knoop H. Severe fatigue after kidney transplantation: a highly prevalent, disabling and multifactorial symptom. Transpl Int. 2013;26(10):1007-15.

15. Zhang R, Jia J, Zhang D, Zhao X. Association between fatigue and depressive symptoms among kidney transplantation recipients: The mediating role of rumination. J Adv Nurs. 2019;75(12):36028.

16. Bossola M, Pepe G, Vulpio C. Fatigue in kidney transplant recipients. Clin Transplant. 2016;30(11):1387-93.

17. Slavish DC, Graham-Engeland JE. Rumination mediates the relationships between depressed mood and both sleep quality and self-reported health in young adults. J Behav Med. 2015;38(2):204-13. 
18. Joormann J, Gotlib IH. Emotion regulation in depression: relation to cognitive inhibition. Cogn Emot. 2010;24(2):281-98.

19. Morin CM, Press TGJS, Disorders S. Insomnia: Psychological Assessment and Management. 1993(6).

20. Nicassio PM, Mendlowitz DR, Fussell JJ, Petras L. The phenomenology of the pre-sleep state: the development of the pre-sleep arousal scale. Behav Res Ther. 1985;23(3):263-71.

21. Espie CA. Understanding insomnia through cognitive modelling. Sleep Med. 2007;8 Suppl 4:S3-8.

22. Amaral AP, Soares MJ, Pinto AM, Pereira AT, Madeira N, Bos SC, et al. Sleep difficulties in college students: The role of stress, affect and cognitive processes. Psychiatry Res. 2018;260:331-7.

23. Li Y, Gu S, Wang Z, Li H, Xu X, Zhu H, et al. Relationship Between Stressful Life Events and Sleep Quality: Rumination as a Mediator and Resilience as a Moderator. Front Psychiatry. 2019;10:348.

24. Tousignant OH, Taylor ND, Suvak MK, Fireman GD. Effects of Rumination and Worry on Sleep. Behav Ther. 2019;50(3):558-70.

25. Smets EM, Garssen B, Bonke B, De Haes JC. The Multidimensional Fatigue Inventory (MFI) psychometric qualities of an instrument to assess fatigue. J Psychosom Res. 1995;39(3):315-25.

26. Chung KF, Yu BY, Yung KP, Yeung WF, Ng TH, Ho FY. Assessment of fatigue using the Multidimensional Fatigue Inventory in patients with major depressive disorder. Compr Psychiatry. 2014;55(7):1671-8.

27. Nolen-Hoeksema S, Morrow J. A prospective study of depression and posttraumatic stress symptoms after a natural disaster: the 1989 Loma Prieta Earthquake. J Pers Soc Psychol. 1991;61(1):115-21.

28. Han XJCJoCP. Chinese Version of Nolen-Hoeksema Ruminative Responses Scale (RRS) Used in 912 College Students:Reliability and Validity. 2009.

29. Buysse DJ, Reynolds CF, 3rd, Monk TH, Berman SR, Kupfer DJ. The Pittsburgh Sleep Quality Index: a new instrument for psychiatric practice and research. Psychiatry Res. 1989;28(2):193-213.

30. Tsai PS, Wang SY, Wang MY, Su CT, Yang TT, Huang CJ, et al. Psychometric evaluation of the Chinese version of the Pittsburgh Sleep Quality Index (CPSQI) in primary insomnia and control subjects. Qual Life Res. 2005;14(8):1943-52.

31. Gao M, Hu J, Yang L, Ding N, Wei X, Li L, et al. Association of sleep quality during pregnancy with stress and depression: a prospective birth cohort study in China. BMC Pregnancy Childbirth. 2019;19(1):444.

32. Gao YQ, Pan BC, Sun W, Wu H, Wang JN, Wang L. Anxiety symptoms among Chinese nurses and the associated factors: a cross sectional study. BMC Psychiatry. 2012;12:141.

33. Reilly-Spong M, Park T, Gross CR. Poor sleep in organ transplant recipients: self-reports and actigraphy. Clin Transplant. 2013;27(6):901-13.

34. Rodrigue JR, Mandelbrot DA, Hanto DW, Johnson SR, Karp SJ, Pavlakis M. A cross-sectional study of fatigue and sleep quality before and after kidney transplantation. Clin Transplant. 2011;25(1):E13-21. 
35. Locke, Jayme, Transplantation EJAJoTOJotASo, Surgeons tASoT. Handbook of Kidney Transplantation. Sixth Edition. G. M. Danovitch (Editor) Wolters Kluwar, 2017, 606 pp. ISBN: 9781496326157. Handbook of Kidney Transplantation. Sixth Edition Handbook of Kidney Transplantation. Sixth Edition. G. M. Danovitch G. M. G. 2018.

36. Hucker A, Bunn F, Carpenter L, Lawrence C, Farrington K, Sharma S. Non-adherence to immunosuppressants following renal transplantation: a protocol for a systematic review. BMJ Open. 2017;7(9):e015411.

37. Tandukar S, Hou S, Yabes J, Ran X, Fletcher M, Strollo P, et al. Does Kidney Transplantation Affect Sleep and Fatigue in Patients With Kidney Disease? Transplant Direct. 2019;5(6):e461.

38. Kompier MA, Taris TW, van Veldhoven M. Tossing and turning-insomnia in relation to occupational stress, rumination, fatigue, and well-being. Scand J Work Environ Health. 2012;38(3):238-46.

39. Querstret D, Cropley M. Exploring the relationship between work-related rumination, sleep quality, and work-related fatigue. J Occup Health Psychol. 2012;17(3):341-53.

40. Querstret D, Cropley M, Fife-Schaw C. Internet-based instructor-led mindfulness for work-related rumination, fatigue, and sleep: Assessing facets of mindfulness as mechanisms of change. A randomized waitlist control trial. J Occup Health Psychol. 2017;22(2):153-69.

41. Abd-Elfattah HM, Abdelazeim FH, Elshennawy S. Physical and cognitive consequences of fatigue: A review. J Adv Res. 2015;6(3):351-8.

42. Rudroff T, Kindred JH, Ketelhut NB. Fatigue in Multiple Sclerosis: Misconceptions and Future Research Directions. Front Neurol. 2016;7:122.

43. Richardson GS. Human physiological models of insomnia. Sleep Med. 2007;8 Suppl 4:S9-14.

44. Sladek MR, Doane LD, Breitenstein RS. Daily rumination about stress, sleep, and diurnal cortisol activity. Cogn Emot. 2020;34(2):188-200.

45. Morin CM, Rodrigue S, Ivers $\mathrm{H}$. Role of stress, arousal, and coping skills in primary insomnia. Psychosom Med. 2003;65(2):259-67.

46. Zhu L, Tao L, Dai J, Liu P. Clinical Status and Correlation of Ruminant Thinking and Sleep Quality in Isolated Population during the COVID-19 Epidemic Journal of Apoplexy and Nervous Diseases. 2020;37(05):409-12.

47. Lazarus R, Folkman S. Stress, Appraisal, and Coping. New York, NY: Springer. 1984.

48. Folkman S, Lazarus RS, Gruen RJ, DeLongis A. Appraisal, coping, health status, and psychological symptoms. J Pers Soc Psychol. 1986;50(3):571-9.

49. Lee H, Yoon JY, Kim I, Jeong YH. The effects of personal resources and coping strategies on depression and anxiety in patients with chronic obstructive pulmonary disease. Heart Lung. 2013;42(6):473-9.

50. Absah I, Rishi A, Talley NJ, Katzka D, Halland M. Rumination syndrome: pathophysiology, diagnosis, and treatment. Neurogastroenterol Motil. 2017;29(4). 
51. de Groot IB, Veen JI, van der Boog PJ, van Dijk S, Stiggelbout AM, Marang-van de Mheen PJ, et al. Difference in quality of life, fatigue and societal participation between living and deceased donor kidney transplant recipients. Clin Transplant. 2013;27(4):E415-23.

52. Zhang HS, Li Y, Mo HY, Qiu DX, Zhao J, Luo JL, et al. A community-based cross-sectional study of sleep quality in middle-aged and older adults. Qual Life Res. 2017;26(4):923-33.

53. Sekercioglu N, Curtis B, Murphy S, Barrett B. Sleep quality and its correlates in patients with chronic kidney disease: a cross-sectional design. Ren Fail. 2015;37(5):757-62.

54. Fresnics AA, Wang SB, Borders A. The unique associations between self-compassion and eating disorder psychopathology and the mediating role of rumination. Psychiatry Res. 2019;274:91-7.

55. Amada NM, Shane JJARR. Mindfulness as a Promoter of Adaptive Development in Adolescence. 2018.

56. Spinhoven P, Klein N, Kennis M, Cramer AOJ, Siegle G, Cuijpers P, et al. The effects of cognitivebehavior therapy for depression on repetitive negative thinking: A meta-analysis. Behav Res Ther. 2018;106:71-85.

57. Hoorelbeke K, Koster EHW. Internet-delivered cognitive control training as a preventive intervention for remitted depressed patients: Evidence from a double-blind randomized controlled trial study. J Consult Clin Psychol. 2017;85(2):135-46.

58. Hirsch CR, Krahe C, Whyte J, Bridge L, Loizou S, Norton S, et al. Effects of modifying interpretation bias on transdiagnostic repetitive negative thinking. J Consult Clin Psychol. 2020;88(3):226-39.

\section{Figures}
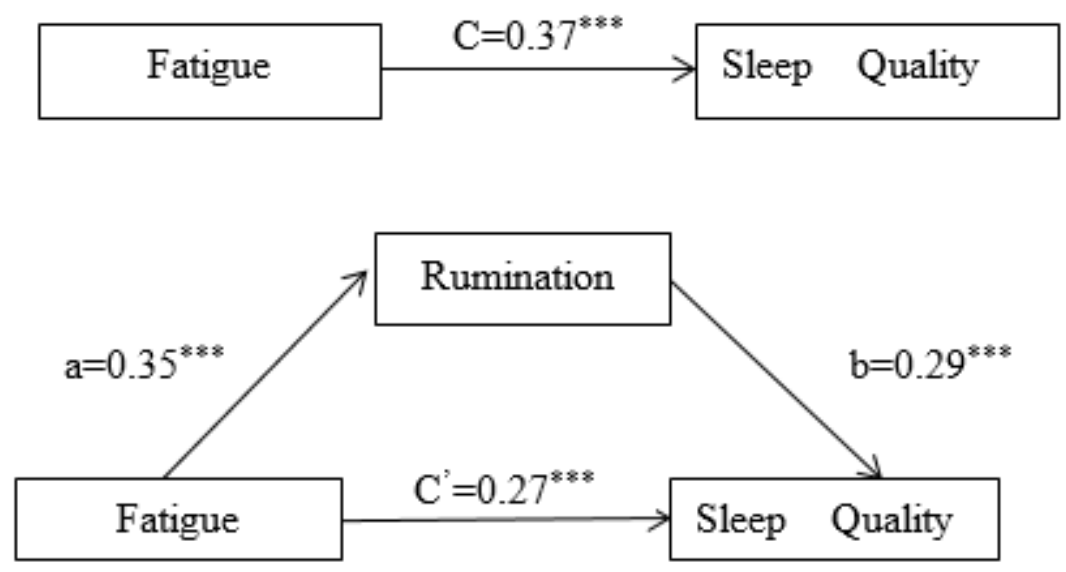

\section{Figure 1}

The mediation model of rumination between fatigue and sleep quality. 